\title{
Clinical implications of antimicrobial resistance
}

\author{
Justin Green
}

Justin Green MA,

$\mathrm{BM}, \mathrm{BCh}, \mathrm{MRCP}$

Specialist Registrar,

Department of

Infectious

Diseases,

Hammersmith

Hospital, London

Clin Med JRCPL

2001;1:408-12

This conference

was held at

the Royal College

of Physicians on

28 February

2001
This enquiry has been an alarming experience, which leaves us convinced that resistance to antibiotics and other anti-infective agents constitutes a major threat to public health, and ought to be recognised as such more widely than it is at present.

House of Lords Select Committee on Science and Technology 7th report (1998)

Are we to enter a 'post-antibiotic era' where control of infectious diseases is threatened by spiralling resistance? We already know that infection with resistant organisms leads to increased morbidity, mortality and cost. Thus a forum to discuss what the practising physician can do was welcomed by the President of the Royal College of Physicians as a positive response to the political and economic alarm initiated by that House of Lords report and those generated subsequently.

\section{How big is the problem?}

Over half of all antibiotic prescriptions are for community acquired respiratory tract infections for which no centralised records are kept and few specimens are sent to the laboratory. Data generated are skewed towards resistant organisms rather than reflecting those prevalent in the community. However, accurate bacteraemia data are available, from the Public Health Laboratory Service (PHLS). This has shown an increase in the incidence of bacteraemia not solely resulting from increased reporting rates or better quality culture systems.

Nationally the proportion of Staphylococcus aureus bacteraemias due to methicillin resistant Staphylococcus aureus (MRSA) has risen from $2 \%$ to $42 \%$ in the last ten years. There is marked inter-trust variation in these rates with some London teaching hospitals reporting levels of MRSA over 50\%. But the type of work that these hospitals carry out is often more specialised and on sicker patients, more at risk of colonisation with multi-resistant organisms. Interpretations of these data are therefore difficult. There has been a progressive loss of antimicrobial effectiveness against gram-positive organisms in general. Resistance in pneumococci to erythromycin has doubled to $11 \%$ in ten years and penicillin resistance is more common in UK isolates, until recently only seen as imported strains. Twenty-five per cent of Enterococcus faecium isolates are resistant to van- comycin, a five-fold rise attained during the 1990s.

Worrying trends in resistance are not limited to gram-positive organisms. Amoxycillin has long been redundant in the empirical treatment of $E$ coli infections, with more than $50 \%$ of community isolates resistant to penicillins. However, gentamicin resistance has trebled to 3\% and is 6\% in Enterobacter spp, common nosocomial pathogens. However, these figures do compare favourably internationally.

Data on prescribing are less easy to obtain and where available are of variable quality. The appropriateness of a prescription is more important information than raw prescribing data. Here information technology can help if we could crosslink pharmacy, laboratory and hospital pathology databases. However, large-scale financial investment will be needed.

\section{Controlling resistance}

In attempting to define ways of controlling resistance in the community an understanding is needed of what the relationship is between prescribing and resistance. Are there other factors affecting resistance other than prescribing habits? Interventions that influence prescribing need evaluating.

At an individual patient level, ample data support the notion that prior use of antibiotics influences future colonisation and infection with more resistant organisms. Previous community antibiotic exposure has been shown to be independently associated with resistance to amoxycillin in Haemophilus influenzae from the sputum of in-patients. However, high rates of trimethoprim-resistant $E$ coli in babies' stool samples were explained by cross-infection occurring in a day care facility. Other confounders to the link between prescribing and infection with resistant strains include extremes of age, urinary tract abnormalities, recent hospitalisation and presence of indwelling catheters.

A four-fold difference in antibiotic prescribing rates between doctors can be demonstrated. General practitioners in 'training practices' consistently write fewer prescriptions per patient per year and use a narrower range of drugs. Reduction in prescribing can be achieved in the community using a combined approach of educational material for patients ('unnecessary antibiotics can be harmful'), as well as doctor education and reappraisal. The $40 \%$ decline 
in antibiotic prescribing for acute bronchitis in one US primary care based study using this approach was not associated with a concomitant increase in the rate of pneumonia. Unfortunately these effects are often short-lived and there is little evidence, as yet, that this approach actually reduces resistance.

Antibiotics used in hospitals are too often unnecessarily broad-spectrum drugs and often given parenterally when orally would suffice. Controlling the prescription of anti-infectives does reap rewards in terms of fewer instances of resistance with the added benefit of cost savings. One London trust saved over $\mathfrak{E 7 7 ,} 000$ in one year by appointing an anti-infective pharmacist providing education, advice and control using antibiotic policies based on local resistance patterns. Constant monitoring allows pre-emptive changes in policy before problems arise. Antibiotic restriction was introduced into a Texan teaching hospital in 1994 with a drop in parenteral antibiotic expenditure by almost a third. Susceptibilities to all $\beta$-lactams and quinolones increased, especially in the intensive care unit. Importantly, there was no deleterious effect on time to drug administration or patient outcome. Targeting prophylaxis in surgery, where a large proportion of hospital antibiotic use occurs, is beneficial. There is no proven benefit of more than 24 hours of use here and the first dose should be given no more than two hours before incision. Future strategies may include educational order forms, computer assisted decision-making and use of information technology to monitor prescribing closely.

Inappropriate use of antibiotics is only part of the problem. Infection control must be part of controlling resistance in hospitals. One in ten inpatients gets a hospital acquired infection in the UK. Isolation and hand hygiene are central to reducing the spread of resistance. Targeting high-risk areas such as intensive care and surgical units is especially important. Specialist nurses, as part of a multidisciplinary team, are invaluable in continually educating and instituting policy. However, infection control must have a higher profile for it to succeed. Unfortunately there are no structures or systems that can efficiently deliver an integrated, co-ordinated infection and antibiotic control policy in most UK hospitals as yet, but with the introduction of clinical governance and accountability of hospital managers for nosocomial infection rates this may well change rapidly.

\section{Resistant pneumoncocci}

Penicillin resistance in pneumococci results from structural changes that decrease affinity of the penicillin binding proteins (PBP) for $\beta$-lactams. Inter-species horizontal transfer of PBP genes, probably from viridans streptococci in the oral flora, confers resistance rather than its development de novo in individual organisms. In the US up to $30 \%$ of pneumococci are penicillin resistant and $11 \%$ are cephalosporin resistant; and rates of resistance are already high in Spain, France, Hong Kong and Saudi Arabia. Penicillin resistance is frequently associated with resistance to many other drugs. Risks for infection with these organisms include recent antibiotic use or hospitalisation, HIV infection, alcoholism and age above 65 years.

Third generation cephalosporins remain the drugs of choice

\section{Conference programme}

- Controlling resistance in the community

Professor P Davey, University of Dundee

I Controlling resistance in hospitals

Dr A Holmes, Imperial College School of Medicine, Hammersmith Hospital, London

How big is the problem?

Dr G Duckworth, CDSC, Public Health Laboratory Service, London

- Pneumonia and meningitis: resistant pneumococci Professor R Finch, University of Nottingham

M tuberculosis and MDR-TB

Professor F Drobniewski, Public Health Laboratory Service, King's College Hospital, London

- Clinical implications of vancomycin resistance

Professor E Rubenstein, Tel-Aviv University, Israel

- The emergence of clinical resistance in viruses other than HIV

Professor P Griffiths, Royal Free and University College Medical School, London

Does anti-fungal resistance matter?

Professor T Rogers, Imperial College School of Medicine, Hammersmith Hospital, London

I Practical implications of resistance to antimalarials Professor P Winstanley, University of Liverpool

- New targets

Professor G Dougan, Imperial College of Science, Technology and Medicine, London

New drugs

Professor E Rubenstein, Tel-Aviv University, Israel

for treatment of pneumococcal meningitis in the UK until sensitivities are known. In published case series there appears to be no difference in mortality or length of stay when using a third generation cephalosporin to treat penicillin-resistant organisms. However, if resistant organisms are isolated a change to vancomycin, probably combined with rifampicin, should be considered; but little data are available. Fully sensitive organisms can be treated with high dose benzyl-penicillin with the possible addition of probenecid to enhance CSF antibiotic levels.

In community acquired pneumonia (CAP) penicillins remain efficacious. Reviews of severe pneumococcal pneumonia show mortality is the same in the penicillin-treated group as in those treated with cephalosporins. This is because concentrations of penicillin in bronchial secretions far exceed those needed to kill resistant organisms. The current British Thoracic Society guidelines for treatment of CAP with a penicillin-based drug (such as amoxycillin or co-amoxiclav), possibly with a macrolide, remain valid. In the US there is a shift towards recommending the newer quinolones such as moxifloxacin but few data exist in the UK as yet. Ciprofloxacin should not be used to treat CAP. No isolation policy guidelines are available for patients with resistant organisms. The pneumococcal vaccine, with up to $93 \%$ efficacy, is underused with only about $30 \%$ of those who warrant vaccination (diabetics, asplenics, those with chronic lung, heart, liver 
and renal disease, HIV, cystic fibrosis and transplants) actually receiving it.

\section{Mycobacterium tuberculosis and MDR-TB}

The annual incidence of tuberculosis worldwide is eight million and rising, causing between two and three million deaths. It is the most important single infectious cause of death on the planet. Although 95-97\% of cases occur in the developing world 6000 new cases are diagnosed every year in the UK and a small and unnecessary percentage of these end in death. Rates of TB notification are higher in urban areas, with London having 3-4 times the national average of cases. This undoubtedly reflects the multicultural society of our inner cities. In immigrant groups TB incidence often reflects that of the country of origin. The upward trend in tuberculosis incidence all over the world is due to a complex interplay between breakdown in surveillance, lack of treatment supervision and increasing HIV infection. More recently this has been complicated by the emergence of multiple drug-resistant tuberculosis (MDR-TB).

MDR-TB is defined as any MTB that is resistant to isoniazid and rifampicin. Treatment is difficult and prolonged and there is a well-documented higher morbidity and mortality rate associated with infection by MDR strains. Ineffective treatment such as non-compliance, incorrect regimens, poor supervision, malabsorption or drug interactions allows selection of organisms with chromosomal mutations conferring resistance. A national laboratory-based surveillance system, MYCOBNET, collates antibiotic susceptibilities, demographics, geographical information and other factors related to drug resistance of MTB isolates. These data are then amalgamated into the World Health Organisation (WHO) global surveillance programme. Prevalence of MDR-TB in the UK is about 1.5\%. In Estonia, one of the worst affected countries, it is $14.1 \%$; and significant increases have been seen in the US and France.

Risks for MDR-TB include previous treatment for MTB, being HIV positive, being hospitalised (nosocomial spread occurs, including to healthcare workers) and living in an area where infection control measures have broken down such prisons and being homeless. Mortality rates for HIV positive patients are $50-90 \%$ and approximately $20 \%$ for HIV negative patients. The single most important factor in lowering the rate of MDR-TB from 19\% to 13\% in New York in 3 years was the revitalisation of the public health system. This allowed the institution of strict supervision of treatment regimens such as direct observed therapy. DOT is twice or thrice weekly administration of drugs in larger individual doses.

Since receiving more than two weeks of appropriate therapy with at least two drugs to which the isolate is susceptible and starting appropriate therapy within four weeks of diagnosis favours a positive outcome in treatment of MDR-TB, more rapid sensitivity testing is needed. Use of broth-based systems has significantly shortened the time to generation of susceptibility data. However, molecular techniques can give results in days. The only one currently available to clinicians is PCR amplification of the rpoB gene. Mutations in this gene are asso- ciated with resistance to rifampicin and are surrogate markers of MDR-TB. Thus other agents can be added in at a much earlier stage. This test can be done on sputum smears that are positive for acid fast bacilli as well as laboratory isolates.

Treatment of MDR-TB is lengthy (18-24 months) and often complicated by side effects of the drugs used. Treatment should always be under specialist supervision and it should not be assumed that a person is non-infectious after two weeks of therapy. The criterion for release from isolation is a minimum of two and ideally three documented negative sputum cultures. Lobectomy or thoracoplasty is sometimes needed in resistant cases where sputum culture conversion cannot be achieved medically. New drugs to treat TB include rifapentene, a long acting rifampicin. Ciprofloxacin has significant anti-mycobacterial properties and is nearly as effective as ethambutol or pyrazinamide. The role of macrolides, such as clarithromycin, is less clear. Exciting work is being done in developing new ways to deliver drugs such as in liposomal form or as depot preparations. Immunotherapy using aerosolised $\gamma$-INF has been used in MDR-TB patients with initial success.

\section{Clinical implications of vancomycin resistance}

The MRSA epidemic has led to a 20-fold increase in the use of vancomycin. Vancomycin resistance first appeared in isolates of coagulase negative staphylococci in 1987. In 1996 a Japanese man was found to have vancomycin-intermediate Staphylococcus aureus (VISA, also known as glycopeptide-intermediate Staphylococcus aureus or GISA) causing his pneumonia. The evolution of GISA strains comes as no surprise but is of enormous international concern. Subsequently GISA isolates have been reported all around the world with the first UK case in 1998. Typical patients have received a prolonged course of vancomycin therapy for an MRSA infection with a poor clinical response, and are often dialysis dependent.

Detection in the laboratory is difficult as the traditional diskdiffusion method is inaccurate. Quantitative methods are better but consume much laboratory time and resources. One possible avenue of compromise is to use agar plates impregnated with a known concentration of vancomycin that selects resistant organisms and to target surveillance to at risk groups such as diabetic patients, intravenous drug users and renal patients on prolonged courses of vancomycin.

The mechanism of how MRSA becomes GISA is not fully elucidated but involves the production of a thickened cellular capsule under vancomycin selective pressure. This prevents the drug from inhibiting transpeptidation between d-alanine moieties in cell wall synthesis. Fortunately they do not yet use mechanisms such as those encoded by the vanA gene which confers high level vancomycin and teicoplanin resistance, such as is found in vancomycin resistant enterococci (VRE). It has, however, been transferred to staphylococci in vitro. GISA exhibit slower growth, increased biofilm formation and better adherence to vascular catheters, as well as up-regulation of expression of penicillin binding proteins. It is easy to see how inappropriate use and poor dosing regimens, seen in nearly two-thirds of 
patients in one retrospective study of vancomycin use, has led to the emergence of this pathogen.

Infection control measures remain the backbone of prevention of spread of GISA. However, when infection is already apparent they do sometimes retain sensitivity to older agents such as co-trimoxazole. There is also in vitro evidence for a possible synergistic mechanism between $\beta$-lactams and vancomycin as they act at different stages of cell wall synthesis. It remains to be seen whether this approach or the use of newer agents such as linezolid $\left(\right.$ Zyvox $\left.^{\circledR}\right)$ and dalfopristin-quinupristin (Synercid ${ }^{\circledR}$ ) will work well in vivo.

VRE can survive on unwashed hands and stethoscopes for 30 minutes or more. The use of vancomycin, cephalosporins and anti-anaerobic antibiotics lead to its increased persistence as part of the gut flora. Crossinfection is important in spread. Antibiotic restriction in specialist units can have a beneficial effect in reducing carriage. However, avoparcin, an animal growth promoter, causes selection for VRE in the GI tract of animals. This, along with the observation that VRE is rarely isolated from the GI flora of non-meat eaters, suggests that control of this organism needs a much wider approach. The Danes prohibited its use in chicken feed and showed a rapid loss of VRE from chicken gut flora over only a three-year period to low levels. Treatment of VRE infections has to be guided by susceptibility to other agents, particularly ampicillin, which should be used if possible. Other agents worth considering include linezolid, dalfopristin-quinupristin, teicoplanin, gentamicin, tetracyclines and carbapenems.

\section{Antiviral resistance}

Aciclovir is tri-phosphorylated by the herpes simplex virus (HSV) thymidine kinase, allowing it to inhibit HSV DNA polymerase. Resistance usually arises by alterations in the thymidine kinase gene leading to reduced enzyme affinity for aciclovir. Occasionally the gene is knocked out completely. Resistant virus and clinical failure is only seen in immunosuppressed patients. Phenotypic and genotypic assays are now commercially available to test isolates for resistance, but before assuming that resistance is the cause of treatment failure one should assess compliance and ensure absorption is adequate. Then a trial of parenteral therapy or use of a newer oral preparation, such as valaciclovir (an aciclovir prodrug with good bioavailability) is indicated. If this fails then viral resistance testing is indicated. Success with vidarabine or foscarnet has been reported in cases of true resistance.

Cytomegalovirus (CMV) can affect many organ systems in the immunosuppressed as well as causing a devastating retinitis in HIV positive patients with low CD4 counts. PCR can detect low levels of viraemia in asymptomatic patients. In both transplant and HIV positive patients detection of CMV viraemia is significantly associated with future clinical disease. The UL 97 gene encodes a phosphotransferase with a similar role to HSV thymidine kinase. Mutations lead to resistance to ganciclovir, the mainstay of CMV treatment. Work from Professor Griffiths' group (Royal Free and University College Medical School,
London) in HIV positive patients with recurrent CMV retinitis showed that over $20 \%$ harboured resistant virus. None of these patients responded to reintroduction of ganciclovir and they needed alternative treatments such as intraocular antivirals, foscarnet or cidofovir. Transplant patients appear to harbour a similar level of resistant virus, with oral ganciclovir prophylaxis acting as a selective pressure due to its low bioavailability.

Drug resistance in influenza strains is currently very low, perhaps reflecting the poor efficacy of currently available drugs. So how will the launch of zanamivir (Relenza ${ }^{\circledR}$ ), a neuraminidase inhibitor that, if given early in infections, can decrease the length and severity of symptoms, influence this? As the symptoms of influenza are non-specific many unnecessary prescriptions will need to be dispensed to protect those at risk, and compliance may be an issue. Although resistant viruses have already been reported they are less transmissible and have a decreased ability to replicate. The current feeling is that resistance will, therefore, not become a problem.

\section{Antifungal resistance}

Systemic fungal infections continue to rise in incidence due to increasing numbers of immunosuppressed patients in the population. There is only a limited repertoire of antifungal drugs available to the clinician. Amphotericin B resistance is still rare and is normally due to intrinsic resistance as seen in some yeasts and moulds. Systemic azole resistance, due to increased expression of cellular efflux mechanisms, was first seen as clinical failure in patients with chronic mucocutaneous candidiasis and HIV positive individuals where repeated treatment courses were needed. Professor Rogers (Imperial College School of Medicine, London) explained that, possibly also because of heavy azole use, the epidemiology of deep-seated candidal infection is changing with azole-resistant species such as Candida glabrata becoming more frequently isolated. One of the problems clinicians face is that in vitro sensitivity tests such as mean inhibitory concentrations (MICs) have been shown to correlate poorly with clinical outcome; and disc susceptibility tests are still only in development. Thus simple measures, such as the removal of indwelling lines upon which biofilms of fungi resistant to drug action form, must be enforced as it has been shown to reduce time to clearance of candidaemia dramatically.

Liposomal forms of amphotericin B are less nephrotoxic and newer azoles such as voriconazole and posaconazole will soon be available. Caspofungin, the first drug in a new class of antifungals called echinocandins, inhibits cell wall growth and is indicated in the treatment of invasive aspergillosis in patients intolerant of or failing on current therapy. It is already available in the US. Trials to evaluate the role of novel antifungal combinations such as fluconazole and flucytosine, or amphotericin and terbinafine, are under way.

\section{Antimalarials}

Falciparum malaria remains a problem worldwide, with $90 \%$ of the burden of disease in Africa where it causes over a million 
deaths every year, largely amongst the young. Resources remain scarce. Thus spraying, widespread provision of bednets and mass chemoprophylaxis campaigns are not financially viable but might be better targeted at high-risk groups such as pregnant mothers to avoid low birth weight babies and fetal loss. Chloroquine resistance is rife, with rates on the Kenyan coast well over $25 \%$. Mass chemoprophylaxis campaigns in the 1950s are responsible for selecting parasites with a point mutation in the $p f m d r$ gene that leads to an up-regulation in efflux of chloroquine from the cell. However, it remains the most common firstline drug as it can effect clinical, if not parisitological cure in the semi-immune. In the non-immune such as the young and foreign travellers it is largely useless. For this reason at least eleven countries have adopted sulfadoxine-pyrimethamine (Fansidar ${ }^{\circledR}$ ), a synergistic combination of two hydrofolate reductase inhibitors, as first-line treatment. Unfortunately it is already a redundant drug in Southeast Asia, where multidrug resistance is a well-established problem.

What might replace chloroquine and sulfadoxinepyrimethamine is not clear, but it must be cheap, safe and easy to administer to outpatients. Quinine, although highly effective, is expensive, tastes awful and is impractical, as it needs to be given for several days. Amodiaquine has an unacceptably high incidence of agranulocytosis and hepatitis. Drugs such as halofantrine, mefloquine, atovaquone-proguanil and the artemisins are too expensive. Combinations of cheaper drugs, eg chlorproguanil and dapsone, that have a low selective pressure for resistance, may offer a way forward.

Quinine remains the drug of choice for treatment of falciparum malaria in the UK. Resistance is at a low level in Africa but is more problematic in Southeast Asia. If used parenterally infusions should be slow to avoid hypoglycaemia. Artemether can be used to treat quinine-resistant parasites but quinine failure is rarely seen.

\section{The future: new drugs and strategies}

The most promising antibiotic advance is linezolid (Zyvox $\left.{ }^{\circledR}\right)$; the first commercially available antibiotic from a new structural class called oxazolidinones that act on the 30s ribosomal subunit, terminating bacterial protein synthesis at an early stage. This novel antibiotic mechanism means cross-resistance is not a problem. It has a similar spectrum and equivalent activity in clinical trials to vancomycin but the added advantage of having an oral preparation that is $100 \%$ bioavailable.

Quinupristin/dalfopristin (Synercid ${ }^{\circledR}$ ) binds the 50s ribosomal subunit, causing premature detachment of incomplete polypeptide chains. It has been successfully used in the treatment of gram-positive infections in vancomycin intolerant and failing patients (up to $92 \%$ of VRE isolates are susceptible). Severe arthralgias and myalgias may necessitate stopping the drug. Other drugs in development include glycylcycline; a tetracycline derivative, daptomycin; a cyclic lipopeptide with good activity against GISA strains and oritavancin; a glycopeptide undergoing phase I trials. The newer quinolones, eg moxi- floxacin, gatifloxacin and levofloxacin, show great promise because their gram-positive cover is far better than ciprofloxacin. Thus they may have a role in the treatment of penicillin-resistant pneumococcal infections.

But is staying one step ahead of organisms the way forward? Traditional targets such as the ribosome, folate pathways, cell division and DNA replication are not proving as fruitful as they once were in producing financially viable, efficacious and safe treatments. Professor Dougan (Imperial College of Science, Technology and Medicine, London), in a keynote speech, described novel approaches using ultramodern molecular techniques such as DNA microarray analysis. This technique can identify genes whose expression is up or down regulated under different environmental stresses and whose products can then be targeted. For example, the presence of isoniazid induces type II fatty acid synthesis within MTB cells. New drugs are now being developed to inhibit the enzymes in this pathway. Sequencing whole pathogen genomes such as Salmonella spp. has already reaped rewards, with the production of a PCR-based system for detecting multidrug resistance in S. typhi. Using 3-D computer modelling drugs can be designed and then synthesised that should inhibit a particular enzyme. A drug that blocks the dehydroquinate synthase stage of the shikimic acid pathway, a group of enzymes found in MTB and malaria species but not humans, has already been designed for use in a TB trial in New York.

\section{Conclusion}

It is clear that we must institute enforceable policy now if we wish to halt the inexorable slide towards the 'post-antibiotic era'. There is ample evidence that strategies exist at all levels to combat the development of resistance should the political and financial will decree it so. However, simpler measures, particularly hand hygiene, will always be the backbone of any war on resistance despite newer solutions showing great promise.

\section{Further reading}

Clinton White Jr A, Atmat R, Wilson J, Cate TR, et al. Effects of requiring prior authorisation for selected antimicrobials: expenditures, susceptibilities and clinical outcomes. CID 1997;25:230-9.

Gonzales R, Steiner J, Lum A, Barrett P. Decreasing antibiotic use in ambulatory practice. JAMA 1999;281:1512-19.

Seaton R, Steinke D, Phillips G, MacDonald T, Davey P. Community antibiotic therapy, hospitalisation and subsequent respiratory tract isolation of Haemophilus influenzae resistant to amoxycillin: a nested casecontrol study. J Antimicrob Chemother 2000;46:307-9.

Bowen E, Emery V, Wilson P, Johnson MA, et al. Cytomegalovirus polymerase chain reaction viraemia in patients receiving ganciclovir maintenance therapy for retinitis. AIDS 1997;12:605-11.

Tenover F. Implications of vancomycin-resistant Staphylococcus aureus. J Hosp Infection 1999;43 (Supplement):S3-7.

Harwell J, Brown R. The drug resistant pneumococcus: clinical relevance, therapy and prevention. Chest 2000;117:530-41.

Website for House of Lords Select Committee on Science and Technology 7th Report:

www.publications.parliament.uk/pa/ld1999798/ldselect/ldsctech/081vii 